\title{
High levels of Ca125 in a patient with large Uterine Fibroid
}

\section{Konstantinos Zacharis, Konstantinos Dafopoulos}

Department of Obstetrics and Gynecology, Faculty of Medicine, School of Health Sciences, University of Thessaly, Larissa, Greece

\section{Corresponding Author}

Konstantinos Zacharis, Department of Obstetrics and Gynecology, University of Thessaly, Biopolis, 41500 Larissa, Greece, Tel.: +30 2413502795, e-mail: zaxarisk@yahoo.com

\begin{abstract}
Uterine leiomyomas, also known as uterine fibroids, are the most common benign tumors of the female genital tract and affect 60 to $80 \%$ of women at their reproductive age. Although elevated tumor markers may be measured in benign gynaecological diseases, the association of uterine fibroids with increased levels of serum cancer antigen 125 (CA125) has not been proven to date. In the present case report we present a rare case of a 21-year-old Caucasian woman attended to our outpatient department with hypermenorrhea and pelvic discomfort that was treated for an enlarged intra-abdominal mass with an abnormally high CA-12 (777.3 U/mL).
\end{abstract}

Key words: CA125, uterine leiomyoma, menstrual

Uterine leiomyomas, also known as uterine fibroids, are the most common benign tumors of the female genital tract and affect 60 to $80 \%$ of women at their reproductive age ${ }^{1}$. Typical clinical presentations include severe menstrual bleeding and lower abdominal pain. Uterine fibroids can also cause infertility, recurrent abortions and preterm labor in cases complicating pregnancy ${ }^{2}$. They are commonly diagnosed by ultrasonography or magnetic imaging resonance. In the current moment, no serum biomarker is capable of monitoring uterine fibroids or differentiating leiomyomas from gynaecological malignancies ${ }^{3}$. Although elevated tumor markers may be measured in benign gynaecological diseases ${ }^{4}$, several studies failed to associate uterine fibroids with increased levels of serum cancer antigen 125 $(\mathrm{CA} 125)^{5-6}$. We hereby report a case of a uterine fibroid with high levels of CA125 prior to surgery.

A 21-year-old Caucasian woman attended to our outpatient department with hypermenorrhea and pelvic discomfort. There was no gynecological disorder on her past medical history. Physical examination on admission revealed a mobile central 14-week size abdominopelvic solid mass and mild generalized pelvic tenderness without sings of peritonitis. Transabdominal ultrasonography showed a 68x62×49 
mm mass developed from uterus, suggesting an intramural uterine fibroid. The presence of large solid mass was also confirmed by pelvic magnetic resonance imaging. At the first consultation complete blood counts, urine analysis and blood biochemistry were all normal. Measurement of CA125 on the 3rd day of the menstrual cycle showed a CA125 serum concentration of $777.3 \mathrm{U} / \mathrm{mL}$ (normal reference ranges: 0-35 U/ml). The levels of carcinoembryonic antigen (CEA) and carbohydrate antigen (CA) 19-9 were within normal range. Patient was taken up for exploratory laparotomy after obtaining informed consent. At laparotomy, a large intramural fibroid with no adhesion to surrounding organs was detected. Additionally, both adnexa appeared normal. After collection of peritoneal washings for cytologic examination, myomectomy was done. Histopathology of the specimen showed a non-malignant leiomyoma of the uterus. Onhematoxylin-eosin staining the tumor appeared typically as leiomyoma and peritoneal washing cytology revealed mesothelial cells and absence of neoplasmatic cells. Post-operative course of the patient was uneventful and elevated serum CA125 level began falling immediately after surgery, and decreased to a normal level, one month after myomectomy.

CA125 is a cell surface glycoprotein present in over $80 \%$ of non-mucinous epithelial ovarian cancers. It is found at low concentration in the serum of healthy males and females ${ }^{7}$. Abnormally high levels of CA125 have been rarely reported in patients with uterine myoma $^{8-10}$. Herein we present a female patient in reproductive age with uterine fibroid and elevated level of CA125 found during menstruation. There are very few findings in the literature, pointing out leiomyomas as cause of CA125 production [8-10]. In our case and in Miyoshi et al. case, serum CA125 was found to be abnormally high during menstruation, indicating that CA125 that leiomyomas produces goes up and down in concordance with the menstruation.
The serum CA125 level dropped rapidly, from 777.3 $\mathrm{U} / \mathrm{mL}$ at first consultation to $363.9 \mathrm{U} / \mathrm{mL}$ two days after the operation. Also levels of CA125 may have been decreasing even before the myomectomy, suggesting that a cyclic production of CA125 by uterine fibroid may have existed, and the peak may have occurred during menstruation.

In conclusion our case endorses that elevated levels of CA125 may be found in benign gynaecological conditions, but the mechanism of this atypical variation of CA125 concentration related to the uterine fibroid is still unknown. Due to rarity of cases, it is important to apply additional clinical data and imaging diagnostic techniques, in order to rule out malignancy of genital tract from differential diagnosis of these patients.

\section{Consent}

Informed consent was obtained.

\section{Conflicts}

The authors declare no conflicts of interest.

\section{References}

1. Laughlin SK, Schroeder JC, Baird DD. New directions in the epidemiology of uterine fibroids. Semin Reprod Med 2010;28:204-17.

2. Bulun SE. Uterine fibroids. N Engl J Med 2013;369 (14):1344-55.

3. Levy G, Hill MJ, Plowden TC, Catherino WH, Armstrong AY. Biomarkers in uterine leiomyoma. Fertil Steril 2013;99:1146-52.

4. Babacan A, Kizilaslan C, Gun I, Muhcu M, Mungen E, Atay V. CA 125 and other tumor markers in uterine leiomyomas and their association with lesion characteristics. Int J Clin Exp Med 2014;7(4):1078-83.

5. Dawood MY, Khan-Dawood FS. Plasma insulinlike growth factor-I, CA-125, estrogen, and progesterone in women with leiomyomas. Fertil Steril 1994;61:617-21. 
6. Dingiloglu BS, Gungor T, Ozdal B, Cavkaytar S, Bilge U, Mollamahmutoglu L. Serum leptin levels in women with uterine leiomyomas. Taiwan J Obstet Gynecol 2007;46:33-7.

7. Bast RC Jr, Badgwell D, Lu Z, Marquez R, Rosen D, Liu J, et al. New tumor markers: CA125 and beyond. Int J Gynecol Cancer 2005 Nov-Dec; 15 Suppl 3:274-81.

8. Ghamande SA, Eleonu B, Hamid AM. High levels of CA-125 in a case of a parasitic leiomyoma presenting as an abdominal mass. Gynecol Oncol 1996 May;61(2):297-8.

9. Ghaemmaghami F, Karimi Zarchi M, Hamedi B.
High levels of CA 125 (over 1,000 IU/ml) in patients with gynecologic disease and no malignant conditions: three cases and literature review. Arch Gynecol Obstet 2007;276:559-61.

10. Miyoshi A, Miyatake T, Komiya S, Mimura M, Nagamatsu M, Yokoi T. Highly Elevated Level of Serum CA125 Produced by a Large Uterine Leiomyoma in a 20-Year-Old Woman. J Clin Gynecol Obstet 2015;4(3):275-8.

Received 07-09-20

Revised 22-09-20

Accepted 28-09-20 